\title{
Moving from Organ Dose to Microdosimetry: Contribution of the Monte Carlo Simulations
}

\author{
Christophe Champion*
}

Laboratoire de Physique Moléculaire et des Collisions; Université de Metz; 1 boulevard Arago; Technopôle 2000; 57078 Metz; champion@sciences.univ-metz.fr; France

\begin{abstract}
When living cells are irradiated by charged particles, a wide variety of interactions occurs that leads to a deep modification of the biological material. To understand the fine structure of the microscopic distribution of the energy deposits, Monte Carlo event-by-event simulations are particularly suitable. However, the development of these track structure codes needs accurate interaction cross sections for all the electronic processes: ionization, excitation, Positronium formation (for incident positrons) and even elastic scattering. Under these conditions, we have recently developed a Monte Carlo code for electrons and positrons in water, this latter being commonly used to simulate the biological medium. All the processes are studied in detail via theoretical differential and total cross sections calculated by using partial wave methods. Comparisons with existing theoretical and experimental data show very good agreements. Moreover, this kind of detailed description allows one access to a useful microdosimetry, which can be coupled to a geometrical modelling of the target organ and then provide a detailed dose calculation at the nanometric scale.
\end{abstract}

Key words: Electron, Positron, Monte Carlo Simulation, Microdosimetry.

\section{INTRODUCTION}

Charged particle track-structure analysis is a useful basis for the understanding of early physical and chemical stages of radiation actions on matter in general. This holds true especially in radiation biology, i.e. in research on the effects of radiation on the biological cell, which has a highly inhomogeneous spatial and chemical structure. Track-structure analysis based on computer simulations requires cross sections as input data in order to describe, step-by-step, all the interactions induced by charged particles with molecules in the matter under consideration (water molecule for biological cells of soft tissues). To date, there are a number of Monte Carlo electron track structure codes in water, which have been developed independently to investigate the microscopic features of ionizing radiation, the ensuing chemical pathways, and the molecular nature of the damages in biomolecular targets (see Uehara et al., 1999 and references therein). A complete model of track structure consists in describing all the elastic and inelastic interactions in terms of total and differential cross sections. The existing codes use a variety of theoretical models and assumptions in treating the physical processes, but are mainly based on semi-empirical fits of experimental singly differential and total cross sections (Uehara et al., 1992). The purpose of this work is to provide useful theoretical cross sections of the most important electronic and positronic

\footnotetext{
${ }^{*}$ Author for correspondence
} 
processes involved in the full slowing-down histories in gaseous water for electrons and positrons with incident energies ranging from several $\mathrm{keV}$ down to a few electron volts.

\section{Monte Carlo basic assumptions}

\section{Biological matter}

Since water is the major component of the human body ( $80 \%$ in mass), the biological medium has been simulated by gaseous water by introducing a density correction of $\rho=1 \mathrm{~g} \cdot \mathrm{cm}^{-3}$. The water molecule wave functions used in this work are those proposed by Moccia (1964), who has expressed each of the five molecular orbital (MO) wave functions of the water molecule in terms of Slater-type-orbital (STO) functions, all centered at a common origin, the oxygen atom. They refer to the equilibrium configurations calculated in the self-consistent field method and agree very well with the experimental geometrical and energetic properties of the water molecule (for more details see Moccia, 1964). In Table 1, the five MO used with their corresponding Ionization Potentials (IP) are reported.

Table 1 - Ionization potentials (IP) of the water molecule.

\begin{tabular}{cc}
\hline Molecular state & Ionization potential \\
\hline${ }^{1} \mathrm{~B}_{1}$ & $\mathrm{IP}_{1}=12.6 \mathrm{eV}$ \\
${ }^{3} \mathrm{~A}_{1}$ & $\mathrm{IP}_{2}=14.7 \mathrm{eV}$ \\
${ }^{1} \mathrm{~B}_{2}$ & $\mathrm{IP}_{3}=18.4 \mathrm{eV}$ \\
${ }^{2} \mathrm{~A}_{1}$ & $\mathrm{IP}_{4}=32.2 \mathrm{eV}$ \\
${ }^{1} \mathrm{~A}_{1}$ & $\mathrm{IP}_{5}=532.0 \mathrm{eV}$ \\
\hline
\end{tabular}

\section{Monte-Carlo principles}

Water molecules are treated as point-like targets and, therefore, (consistent also with the gas phase approximation) any potential energy associated with an ionization or excitation event is assumed locally deposited. The transport simulation, in essence, comprises a series of Monte Carlo (MC) sampling steps which determine: (i) the distance to the next interaction, (ii) the type of interaction that occurred at the point selected in (i), and (iii) the energy and direction of the resultant particles according to the type of interaction selected in (ii) (see Fig. 1). Thus, once the initial parameters of the primary particle are defined, i.e. its energy and its type $\left(Z_{\text {inc }}=+1\right.$ for the positron and $Z_{\text {inc }}=-1$ for the electron), the code determines the free path traveled by direct random sampling according to the sum of all the total interaction cross sections relative to all the processes included in the simulation. The particle is then transported to its new position. By again applying direct $\mathrm{MC}$ sampling according to the relative magnitude of the individual total interaction cross sections (elastic scattering, ionization, excitation and Positronium formation cross sections) the type of collision is randomly determined:

If the interaction is elastic, then, by applying the random MC sampling according to the singly differential cross sections, the scattered direction is determined at the appropriate electron incident energy $E_{i n c}$, which remains quasi unchanged since the energy transfer induced during the elastic scattering is very small (of the order of meV).

In the case of ionization, then, by applying the random MC sampling according to the singly differential cross sections, the kinetic energy of the ejected electron $E_{e}$ is determined. Following the choice of $\mathrm{E}_{\mathrm{e}}$, application of direct MC sampling according to the relative magnitude of the partial total ionization cross sections (at $\mathrm{E}_{\mathrm{e}}$ ) furnishes the ionization potential $\mathrm{IP}_{\mathrm{j}}$. The particle energy is reduced by $\mathrm{E}_{\mathrm{e}}+\mathrm{IP}_{\mathrm{j}}$, while the ejected and scattered directions are determined according to the fivefold and triply differential cross sections, respectively (denoted 5DCS and 3DCS in the following). Indeed, all the geometrical parameters $\theta_{\mathrm{e}}, \varphi_{\mathrm{e}}, \theta_{\mathrm{s}}$ 
and $\varphi_{\mathrm{s}}$ are randomly sampled from successive integrations of the 5DCS defined by $\sigma^{(5)}=\mathrm{d}^{5} \sigma / \mathrm{d} \Omega_{\mathrm{s}} \mathrm{d} \Omega_{\mathrm{e}} \mathrm{dE}$ e where $\Omega_{\mathrm{s}}$ is the scattered direction, $\Omega_{\mathrm{e}}$ the ejected direction and $\mathrm{E}_{\mathrm{e}}$ the energy transfer. Then, the particular ionization potential $\mathrm{IP}_{\mathrm{j}}$ is considered as locally deposited energy, except for inner-shell ionization for which Auger electrons are taken into account and assumed to be isotropically emitted with a kinetic energy $\quad \mathrm{E}_{\text {Auger }} \quad\left(\equiv \mathrm{IP}_{5}-(2 * 32.2)\right), \quad$ i.e. $\mathrm{E}_{\text {Auger }}=467.6 \mathrm{eV}$. In this case, the local energy deposit is then $2 * 32.2=64.4 \mathrm{eV}$.
If excitation is decided, direct MC sampling is applied according to the relative magnitude of all the partial excitation cross sections for determining the transition level $n$. The particle energy is reduced by $\mathrm{E}_{n}$ while the incident direction remains unaltered. Similar to the case of ionization, the particular excitation potential $\mathrm{E}_{n}$ is considered as locally deposited. Moreover, according to experimental results, it is assumed that impact excitation induces no angular deflection.

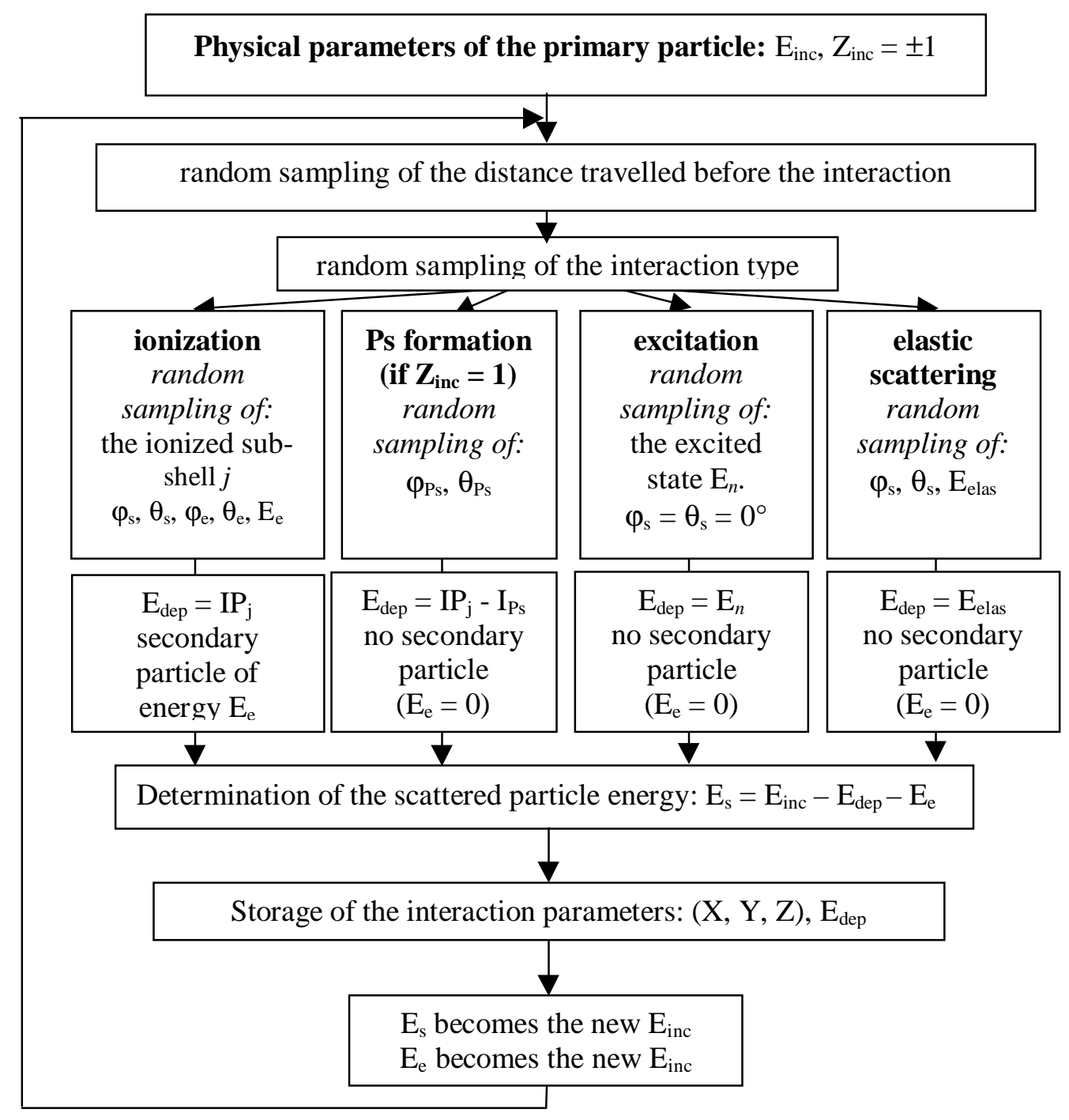

Figure 1 - Schematic representation for the Monte Carlo simulation of positron and electron transport in water. This sequence is followed at each energy step and for all the primary positrons and secondary particles (positrons and electrons). 
If, finally, Positronium formation is decided (only if $E_{\text {inc }} \geq I_{1}-I_{P s}$, where $I_{P s}$ corresponds to the Positronium binding energy: $\mathrm{I}_{\mathrm{Ps}}=6.8 \mathrm{eV}$ ), electron capture by positron impact can occur to form an electron-positron pair called Positronium and denoted Ps. Similarly to the case of ionization, the molecular subshell concerned by the capture process according to the relative magnitude of all the partial total capture cross sections is first determined. Concerning the ejected direction of the Positronium, it can be selected from the corresponding double differential cross sections, but for computing velocity, it is assumed that Positronium formation induces no angular deflection and the Positronium is ejected in a direction collinear to that of the incident positron. Finally, the quantity $\left(\mathrm{IP}_{\mathrm{j}}-\mathrm{I}_{\mathrm{Ps}}\right)$ is assumed locally deposited, whereas the kinetic energy $\mathrm{E}_{\mathrm{Ps}}$ of the Positronium is determined from kinematical considerations, thanks to: $E_{i n c}+I_{P s}=E_{P s}+I_{j}$. All these steps are consecutively followed for all resultant particles until their kinetic energy falls below the predetermined cut-off value $\left(\mathrm{E}_{\mathrm{th}}=7.4 \mathrm{eV}\right)$. Sub-threshold electrons and positrons are then assumed to deposit their energy where they are created. Indeed, in this code, electrons and positrons with $\mathrm{E}_{\text {inc }}<\mathrm{E}_{\text {th }}$ are not followed. These slow electrons essentially induce excitations and elastic collisions whose total cross section becomes very large (about $20.10^{-16} \mathrm{~cm}^{2}$ ), leading to a mean free path smaller than $1 \mathrm{~nm}$. The residual energy of such "dead particles" is deposited in a single step at a single point. The assumption that these slow particles stay where they have been created introduces uncertainties smaller or of the order of $1 \mathrm{~nm}$ in the map of the energy deposits scored at the end of the physical stage following the irradiation $\left(t<10^{-15} \mathrm{~s}\right)$. Approximately $15 \%$ of total energy is deposited in this way. The energy of these "dead particles" may substantially affect the results regarding the clustering energy volumes for only very small target volumes and small energies. In contrast, for longer energies and volumes, the exclusion of these "dead particles" should have little effect, other than reducing the energy axis by $15 \%$ (Nikjoo and Goodhead, 1991). In fact, electrons and positrons with energy below $\mathrm{E}_{\text {th }}$ should be followed by using other techniques (diffusion equation, etc.), but the aim of this MC simulation is to determine initial conditions for subsequent chemical, biological and medical reactions at a very short time scale $\left(\mathrm{t}<10^{-15} \mathrm{~s}\right)$.
Under these conditions, the code is able to provide the coordinates of all the interaction events by way of row data, as well as the type of collision together with the energy loss, the energy deposited at each interaction point and the kinetic energy of the resultant particle(s) in the case of inelastic collision. Interactions of electrons and positrons with water molecules can be divided up into elastic collisions, leading to negligible energy transfers, but with very large momentum transfers (i.e. very large angular deflections), and inelastic collisions (ionization, excitation and capture) leading to the slowing down of the particles and, to a lesser extent, to their angular deflection. Note that the multiple electronic processes are not taken into account in this code since they become important only for incident energies of several keV (Gryzinski, 1965), i.e. for a minor part of the particles studied in these simulations. Similarly, the contribution of Bremsstrahlung to the electronic and positronic slowing-down because of its minor influence in the energy range considered here $\left(E_{\text {inc }}<1 \mathrm{MeV}\right)$ has been neglected.

\section{Positron and electron interaction cross sections}

To describe following-up of electrons and positrons in water, it is essential to have access, step by step, interaction after interaction, to the complete kinematics of all the collisions induced by the primary particle and by all the secondary particles generated. For this purpose, a large set of differential and total cross sections corresponding to the different type of interactions induced by the charged particles in the medium is absolutely necessary. In the following section, all these cross sections whose calculation has been performed in the quantum-mechanic framework are described.

\section{Elastic scattering}

Although the water molecule is tri-atomic, the charge distribution is quasi spherical and governed mainly by the "central" oxygen atom. In fact, the water molecule has an important dipole moment of 1.86 Debye, which is very significant in scattering of electrons at low incident energy $\left(\mathrm{E}_{\text {inc }}<300 \mathrm{eV}\right)$. Therefore, the perturbation potential of the water molecule can be approximated by a spherically symmetric potential $\mathrm{V}(\mathrm{r})$ composed of three different terms: the first representing the static contribution, called $\mathrm{V}_{\text {stat }}(\mathrm{r})$ and deduced from the 
water molecule wave functions; and two fine effect terms - the polarization and the exchange terms introduced from model potentials called $\mathrm{V}_{\text {pol }}(\mathrm{r})$ and $\mathrm{V}_{\text {exc }}(\mathrm{r})$, respectively.

Polarization effects are described thanks to a polarization potential which offers the well known asymptotical limit at large distance proportional to $\left(\alpha / \mathrm{r}^{4}\right)$, where $\alpha$ is the polarizability of the water molecule ( $\alpha=9.7949$ a.u.).

Exchange effects are treated via a model potential deduced from the "Free Electron Gas model" introduced by Slater (1960) and used by Hara (1967).

The calculated differential cross sections are in very good agreements with a large set of experimental data (Champion, 2003). By integrating these differential cross sections over the scattered solid angle $\Omega_{\mathrm{s}}$, the total cross sections, which are reported in Fig. 2, are obtained.

\section{Ionization}

In the major part of the existing numerical simulations of electron track-structure in water, ionization is treated via semi-empirical expressions deduced from experiments. Very recently, differential and total ionization crosssection calculations were developed (Champion, 2003) within the Distorted Wave Born Approximation framework (DWBA), where the incident and scattered (fast) electrons are described by a plane wave function, whereas the ejected (slow) electron is described by a distorted wave function. From five-fold differential cross sections, the triply, singly differential and then total cross sections have been successfully calculated. Very good agreements have been found with available experimental measurements for a large range of incident energies $\left(\mathrm{E}_{\text {inc }}=10 \mathrm{eV}-10 \mathrm{keV}\right)$.

It is important to note that this Monte Carlo code appears to be the first one giving a complete theoretical description of the ionization process without any adjustable parameter.

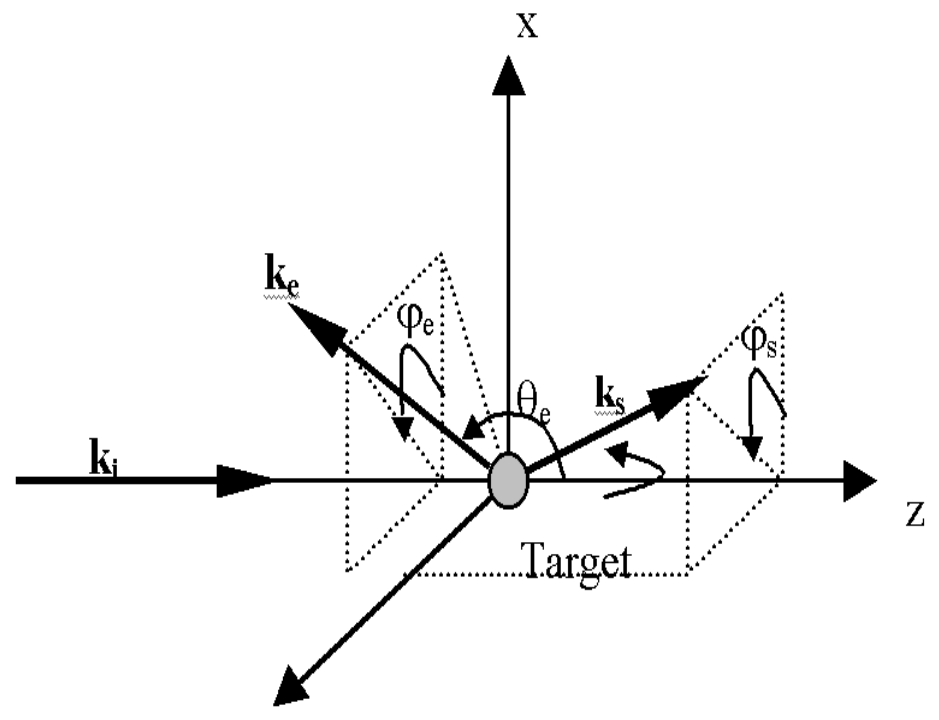

Figure 2 - Reference frame of the ionizing collision of a water target. $\mathbf{k}_{\mathbf{i}}, \mathbf{k}_{\mathbf{s}}$, and $\mathbf{k}_{\mathbf{e}}$ represent the wave vectors of the incident, scattered and ejected electrons, respectively. The corresponding polar and azimuthal angles are denoted $\theta_{\mathrm{s}}, \varphi_{\mathrm{s}}$ and $\theta_{\mathrm{e}}, \varphi_{\mathrm{e}}$, respectively.

\section{Excitation}

Excitation includes all the processes that modify the internal state of the molecule, without emission of electrons. These processes include in particular: i) Electronic transitions towards Rydberg states or degenerate states $\left(\tilde{\mathrm{A}}_{1} \mathrm{~B}_{1}, \widetilde{\mathrm{B}}_{1} \mathrm{~A}_{1}\right.$, diffuse band) 
ii) Dissociative attachment leading to the formation of negative ions

iii) Dissociative excitation, leading to excited radicals $\left(\mathrm{H}^{*}, \mathrm{O}^{*}\right.$ et $\left.\mathrm{OH}^{*}\right)$ and in a minor part

iv) Vibrational and rotational excitations.

All these processes give a non-negligible contribution to the energy deposit processes. To take into account all the processes listed $i$ ), ii) and (1972) were used, whereas dissociative attachment process were treated via the expressions of Green and Stolarski (1972). Moreover, following some experimental results (Compton and Christophorou, 1967), it is assumed that excitation induces no angular deflection.

\section{Positronium formation}

Because of its positive charge, the positron has, contrary to the electron, the possibility of capturing one of the target electrons, leading in the final channel of the reaction to the formation of a
Positronium (neutral electron-positron pair). In these last years, collisions of positrons impacting atomic and/or molecular targets have known a growing interest as a result of the development of high-intensity positron sources and, especially, the systematic use of medical imaging in medicine via the Positron Emission Tomography (PET). Very recently, the Positronium formation for positron impinging water molecule was studied and a theoretical approach within the framework of the independent electron model (IEM) was proposed, providing differential and total cross sections for the electron capture process. This study permits, for the first time, the evaluation of the relative contribution of the Ps formation in the inelastic processes induced by the positron in liquid water. Total cross sections for Positronium formation have been calculated by using the partial wave technique already presented in (Champion, 2003). All the total cross sections presented above are reported in Fig. 3.

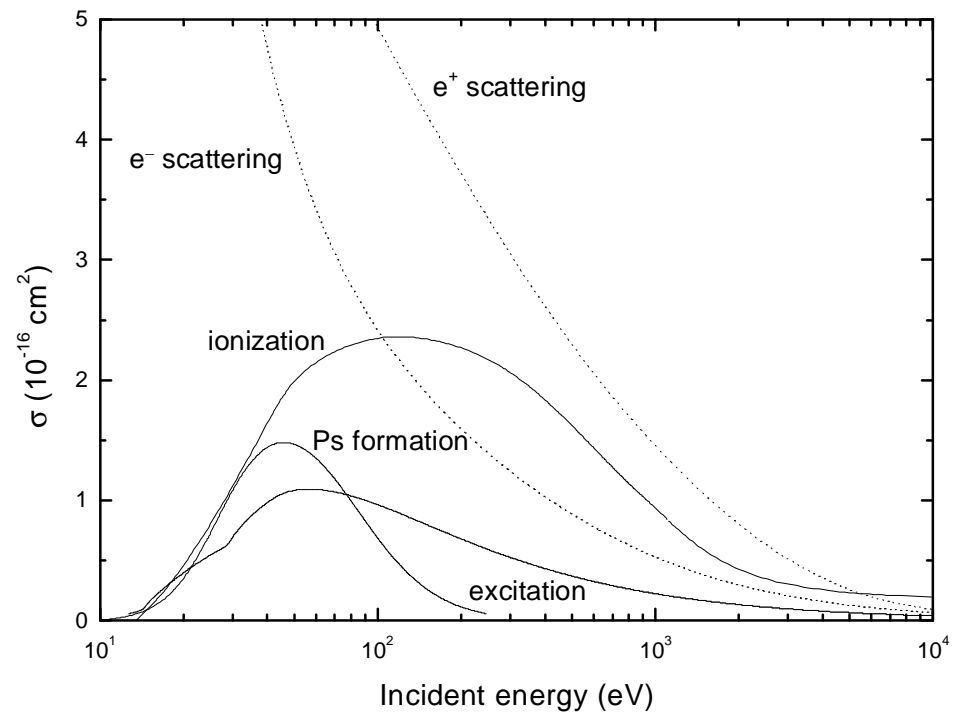

Figure 3 - Total cross sections for elastic (dotted line) and inelastic (solid line) electron and positron-water molecule collisions.

\section{Application to microdosimetry}

As noted before, all the cross sections presented in this work are, strictly peaking, appropriate only for water in the vapor phase where the molecules act largely independently. Simple density scaling to account for condensed-state effects is obviously an oversimplification. Differences between the vapor and the liquid phases in terms of the single- collision energy-loss spectrum, resulting from differences in oscillator strength, and the delocalization of energy transfers, due to collective motion of the target electrons, have been discussed in detail elsewhere (Inokuti, 1991). And in some cases, they have been incorporated into Monte Carlo codes (Turner et al., 1988). However, the justification for continuing research and use of 
vapor-based differential and total cross sections is probably best put forward in a relatively recent ICRU report (1996) where the conclusion is drawn that all attempts at establishing condensed state singly differential cross sections are based on minimal experimental verification. This observation is in sharp contrast with the vapor phase where, for the most part, well-tested experimental data do exist. For example, probably the most recent and comprehensive study on liquid water inelastic cross sections by Dingfelder et al. (1998) is still based on a simple extrapolation of optical data to non-zero momentum transfers by means of the impulse approximation and an analytical function for the Generalized Oscillator Strength (GOS). The degree of accuracy of such an extrapolation function for liquid water has not yet been rigorously investigated in terms of the cross section because of the lack of appropriate data for this medium.

Fig. 4 depicts a 2D reconstruction of a track segment for a $5 \mathrm{keV}$ electron (left panel) and a 5 $\mathrm{keV}$ positron (right panel) in liquid water produced by our MC code with the positions of all the energy deposits (inelastic collisions).
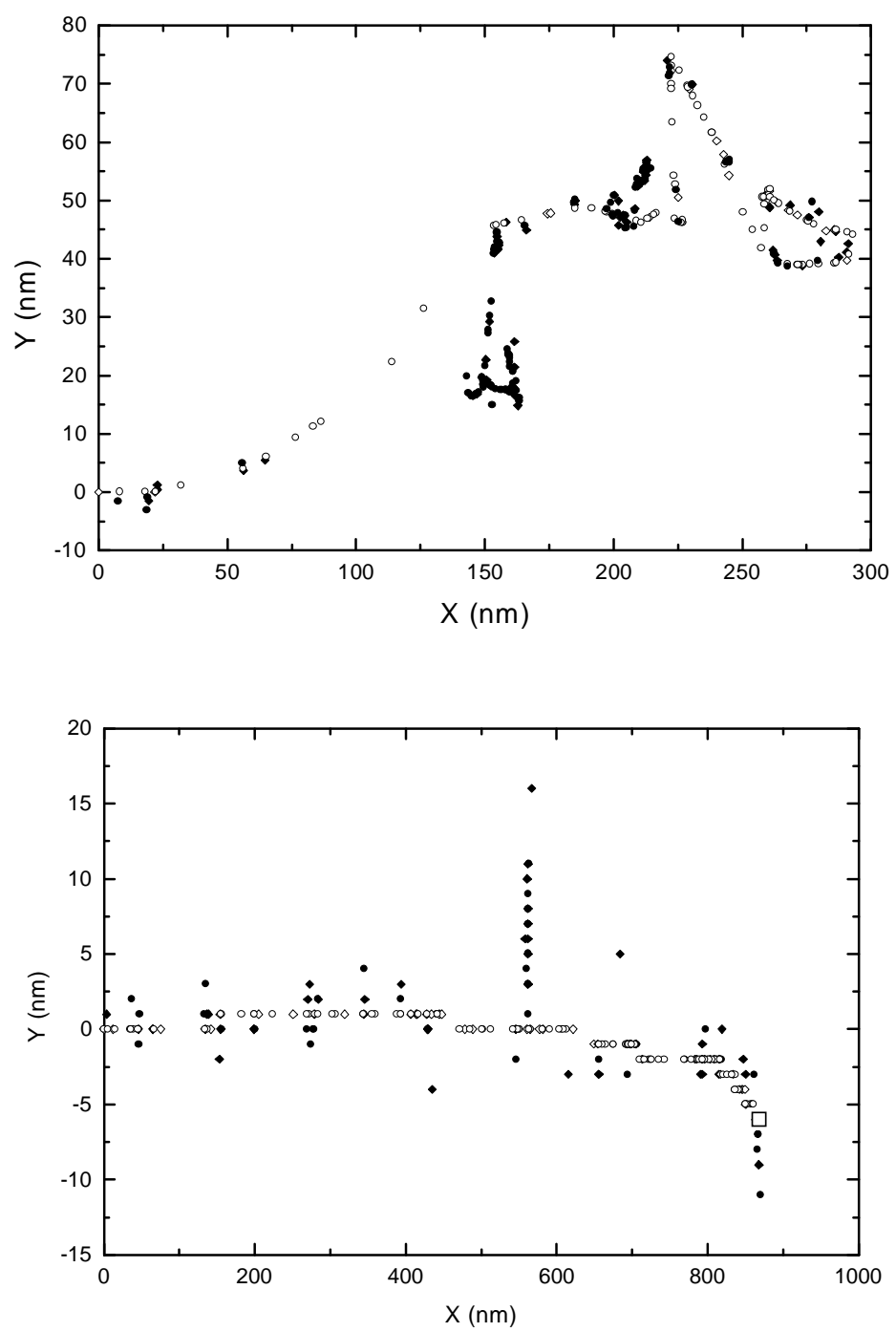

Figure 4 - 2D plot for a $5 \mathrm{keV}$ electron and a $5 \mathrm{keV}$ positron track in liquid water. Primary and secondary interactions are represented by open and solid symbols, respectively (circles for ionizations, diamonds for excitations and a square for the Positronium formation) 
The starting point is located at $(\mathrm{X}=0 ; \mathrm{Y}=0 ; \mathrm{Z}=$ $0)$, and the initial direction is $(\mathrm{Y}=0 ; \mathrm{Z}=0)$. The positions of all the interactions induced by the primary and the secondary particles are explicitly marked. In the left panel, the incident electron induces 103 inelastic interactions (82 ionizations and 21 excitations), whereas the secondary electrons induce 177 inelastic interactions (109 ionizations and 68 excitations). In the right panel, the incident positron induces 136 inelastic interactions (100 ionizations, 35 excitations and 1 Positronium formation), whereas 132 inelastic interactions have been induced by the secondary electrons (60 excitations and 72 ionizations).

\section{CONCLUSION}

A complete numerical simulation that calculates the electron and positron trajectories in water without any adjustable parameter was developed. The calculations were performed in the quantum-mechanic framework, and the overall computer time remains reasonable even for simulating irradiated volumes of few millimeters. The energy loss properties of electrons and positrons will be compared in terms of range, energy loss and stopping power. The energetic cartography finally obtained would be a powerful tool for dose calculation in micrometric biological volumes to evaluate with a high degree of precision the real dose deposited in the irradiated targets.

\section{RESUMO}

Quando células vivas são irradiadas por partículas carregadas, ocorre uma grande variedade de interações, o que leva a uma modificação profunda do material biológico. Para entender a delicada estrutura da distribuição microscópica dos depósitos de energia, as simulações de Monte Carlo são particularmente adequadas. Entretanto, o desenvolvimento destes códigos necessitam de amostras representativa de interações perfeitas para todos os processos eletrônicos: ionização, excitação, formação de positrônico (para pósitrons incidentes) e mesmo espalhamento elástico. Nessas condições, nós desenvolvemos recentemente um código Monte Carlo para elétrons e pósitrons em água usada posteriormente para simular o meio biológico. Todos os processos são estudados detalhadamente via seções de choque calculada usando métodos de ondas parciais. Comparações com dados experimentais e teóricos mostram boa concordância. Além disto, esta descrição detalhada nos permite acessar esta útil microdosimetria, a qual pode ser acoplada a modelo geométrico de órgãos alvo e então fornece um cálculo detalhado da dose em escala nanométrica.

\section{REFERENCES}

Champion, C., Hanssen, J., and Hervieux, P. A. (2002), Electron impact ionization of water molecule. J. Chem. Phys., 117, 197-204.

Champion, C. (2003), Theoretical cross sections for electron collisions in water: structure of electron tracks. Phys. Med. Biol. 48, 2147-2168.

Compton, R. N., and Christophorou, L. G. (1967), Negative-ion formation in $\mathrm{H}_{2} \mathrm{O}$ and $\mathrm{D}_{2} \mathrm{O}$. Phys. Rev., 154, 110-116.

Dingfelder, M., Hantke, D., Inokuti, M., and Paretzke, H. G. (1998), Electron inelastic-scattering cross sections in liquid water. Radiat. Phys. Chem., 53, 1-18.

Goodhead, D. T. (1999), Comparison and assessment of electron cross sections Monte Carlo track structure codes. Radiat. Res., 152, 202-213.

Green, A. E. S., and Stolarski R. S. (1972), Analytic models of electron impact excitation cross sections. Journal of atmospheric and terrestrial physics, 34, 1703-1717.

Gryzinski, M. (1965), Classical theory of atomic collisions. I. Theory of inelastic collisions. Phys. Rev. B, 138, A336-A358.

Hara, S. (1967), The Scattering of Slow Electrons by Hydrogen Molecules. J. Phys. Soc. Japan, 22, 710718.

ICRU, (1996), Secondary electron spectra from charged particle interactions. In ICRU Report 55, (Bethesda, MD: ICRU).

Inokuti, M. (1991), How is radiation energy absorption different between the condensed phase and the gas phase? Radiat. Eff. Def. Sol., 117, 143162.

Moccia, R. (1964), One-center basis set SCF MO's. III. $\mathrm{H}_{2} \mathrm{O}, \mathrm{H}_{2} \mathrm{~S}$, and $\mathrm{HCl}$. J. Chem. Phys., 40, 21862192. 
Nikjoo, H. and Goodhead, D. T. (1991), Track structure analysis illustrating the prominent role of low-energy electrons in radiobiological effects of low-LET radiations. Phys. Med. Biol., 36, 229-238.

Uehara, S., Nikjoo, H., and Olivero J. J., Stagat R. W., and Green A. E. S. (1972), Electron deposition in water vapor with atmospheric applications. Journal of Geophysical Research, 77, 4797-4811.

Slater, J.C. (1960). In Quantum Theory of Atomic Structure. McGraw-Hill Ed. Vol. 2, New York.

Turner, J. E., Hamm, R. N., Wright, H. A., Ritchie, R. H., Magee, J. L., Chatterjee, A., and Bolch, W. E. (1988), Studies to link the basic radiation physics and chemistry of liquid water. Radiat. Phys. Chem., 32, 503-510.

Uehara, S., Nikjoo, H., and Goodhead, D. T. (1992), Cross-sections for water vapour for the Monte Carlo electron track structure code from $10 \mathrm{eV}$ to the MeV region. Phys. Med. Biol., 37, 1841-1858.

Received: June 08, 2005;

Revised: July 14, 2005; Accepted: August 01, 2005. 